\title{
Tunneling spectroscopy of heavily underdoped crystals of $\mathrm{Bi}_{2} \mathrm{Sr}_{2} \mathrm{CaCu}_{2} \mathrm{O}_{8+\delta}$
}

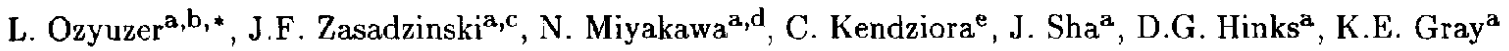 \\ ${ }^{a}$ Materials Science Division, Argonne National Laboratory, Argonne, IL 60439, USA \\ ${ }^{b}$ Department of Physics, Izmir Institute of Technology, TR-35230 Izmir, Turkey \\ 'Illinois Institute of Technology, Chicago, IL 60616, USA \\ ${ }^{\mathrm{d}}$ Department of Applied Physics, Science University of Tokyo, Tokyo 162-8601, Japan \\ eNaval Research Laboratory, Washington, D.C. 20375, USA
}

Crystals of $\mathrm{Bi}_{2} \mathrm{Sr}_{2} \mathrm{CaCu}_{2} \mathrm{O}_{8+\delta}$ with optimal $\mathrm{T}_{c}=95 \mathrm{~K}$ have been underdoped using two different methods and the superconducting gaps have been abtained by tunneling. In some cases, three different tunneling geometries have been utilized: point contact, STM and break junctions. The first doping method involves control of the oxygen content by annealing in various partial pressures of oxygen. These crystals exhibit a narrow spread of gap values over a wide doping range from overdoped $\left(T_{c}=56 \mathrm{~K}\right)$ to underdoped with $T_{c}=70 \mathrm{~K}$. However, for underdoped crystals with $\mathrm{T}_{c}$ midpoints in the range $25 \mathrm{~K} \cdot 63 \mathrm{~K}$, there is a dramatic increase in the spread of gap values which may signal the development of static phase separation of either chemical or electronic origin. To avoid possible chemical phase separation, we have explored another doping procedure which incorporates Dy substitution on the $\mathrm{Ca}$ site. These crystals exhibit a relatively narrow superconducting transition width and some preliminary tunneling spectra will be presented.

As the struggle to understand the origin of high $\mathrm{T}_{c}$ superconductivity continues, the doping dependence of various physical properties has become increasingly important. One of the fundamental superconducting parameters, the energy gap magnitude, follows $\mathbf{T}_{\mathcal{c}}$ in the overdoped regime of $\mathrm{Bi}_{2} \mathrm{Sr}_{2} \mathrm{CaCu}_{2} \mathrm{O}_{8+\delta}(\mathrm{Bi}-2212)$ indicating conventional behavior, however the underdoped phase shows that the energy gap magnitude increases with decreasing hole concentration even as $\mathrm{T}_{c}$ drops to $70 \mathrm{~K}$ from its optimal value of 95 $K$. Moreover, the energy gap more closely follows the pseudogap temperature, $T^{*}[1]$.

In this paper, we present tunneling spectroscopy measurements of heavily underdoped samples with $T_{c}$ as low as $25 \mathrm{~K}$. It will be shown that for underdoped crystals with $T_{c}$ midpoints in

* ozyuzer@anl.gov, work supported by US-DOE BES-MS, W-31-109-ENG-38 and NSF Office of Science and Technology Centers, DMR 91-20000. the range of 25-63 K, there is a dramatic increase in the spread of gap values making it difficult to ascertain any trends.

The single crystals of $\mathrm{Bi}-2212$ are prepared by two different methods, either self-flux or floating zone, and no appreciable difference between crystals has been observed [1]. The crystals are underdoped by annealing in various low partial pressures of oxygen. Dy-doped samples are prepared by replacing ten percent of the $\mathrm{Ca}$ with Dy. Tunneling experiments have been conducted by a system described elsewhere [2] and detailed description of making SIN and SIS junctions can be found in ref. [3].

Figure 1 shows the energy gap versus hole concentration phase diagram for Bi-2212 from SIS and SIN tunneling spectroscopy. Hole concentration, $\mathrm{p}$, is obtained from empirical formula, $\mathrm{T}_{c} / \mathrm{T}_{c}^{\max }=1-82.6(\mathrm{p}-0.16)^{2}$, where $\mathrm{T}_{c}^{\max }=95 \mathrm{~K}$ for Bi-2212. Full circles in Fig. 1 are previously pub- 


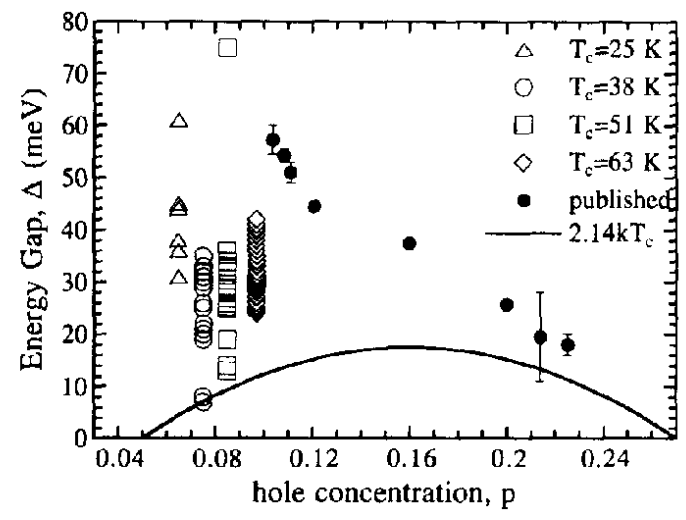

Figure 1. Phase diagram of energy gap versus hole concentration from tunneling spectroscopy. Tunneling measurements are taken at $4.2 \mathrm{~K}$.

lished results corresponding to data from ref. [1] and indicate that the energy gap magnitude has a very narrow spread as shown by the error bars. For example, the point for optimal doping corresponds to about 20 junctions of both SIN and SIS type. Such a narrow spread allows trends to be discerned and it can be seen that the energy gap exhibits a monotonic dependence on doping approaching the $\mathrm{BCS}$ prediction for $\mathrm{d}$-wave superconductors, $2.14 \mathrm{kT}_{c}$ with increasing hole concentration. The data for heavily underdoped crystals with $\mathrm{T}_{c}=25,38,51$, and $63 \mathrm{~K}$ are the new results of this work and show an enormous increase in the distribution of gap values. Each data point is obtained from a different junction and examples of some spectra are shown in Fig. 2. Although, the energy gap magnitude varies considerably from junction to junction for the same $T_{c}$, the main difference is the energy scale and not the spectral shape. This dramatic increase in the spread of gap values for samples with $T_{c}<70 \mathrm{~K}$ may signal the development of static phase separation of either chemical or electronic origin. Since point contact tunneling is a local probe, the local inhomogeneities of the sample may cause the large spread of $\Delta$ magnitudes in the tunneling spectra of heavily underdoped Bi-2212.

One way of examining this spread is to take a horizontal cut through the underdoped region of Fig. 1, that is, to consider junctions which

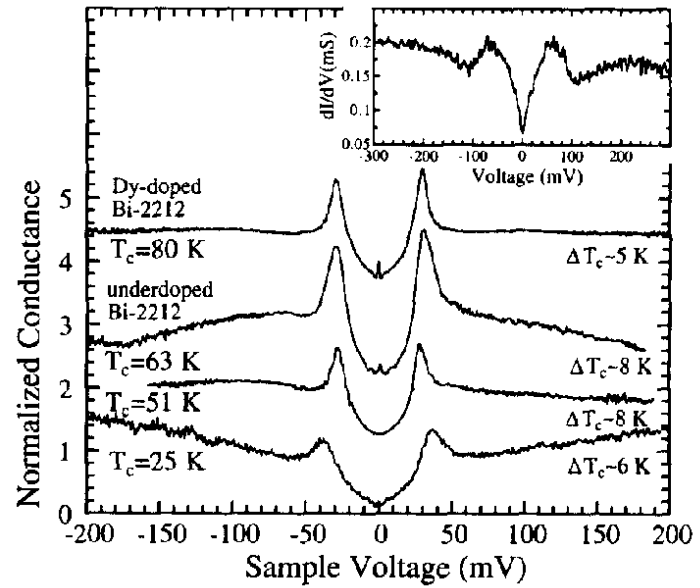

Figure 2. Tunneling conductances of underdoped Bi-2212 and Dy-doped Bi-2212 SIN junctions at $4.2 \mathrm{~K}$ from point-contact measurements. Data are shifted vertically for clarity. The inset shows largest gap obtained from $\mathrm{T}_{c}=25 \mathrm{~K}$ samples.

exhibit a similar gap magnitude despite the different doping and $T_{c}$ values. The bottom three curves of Fig. 2 shows representative tunneling conductances of SIN junctions with a gap parameter near $35 \mathrm{meV}$ at $4.2 \mathrm{~K}$ for three different oxygen-controlled hole concentrations. The top curve is the Dy-doped Bi-2212 with $\mathrm{T}_{c}=80$ $K$. From top curve to the bottom there is a decreasing hole concentralion. As is seen with dccreasing hole concentration quasiparticle peaks are less pronounced. However, tunneling conductances preserve their generic features such as dip at the occupied part of the density of states and hump feature. The spectral weight of quasiparticle peaks are consistent with ARPES experiments that it decreases with decreasing doping. These curves seem to suggest that there is another parameter in addition to hole concentration which is affecting the gap magnitude, perhaps disorder.

\section{REFERENCES}

1. N. Miyakawa et al., Phys. Rev. Lett. 83 (1999) 1081.

2. L. Ozyuzer et al., Cryogenics 38 (1998) 11.

3. Y. DeWilde et al., Phys. Rev. Lett. 80 (1998) 153. 